\title{
Transplanted Olfactory Ensheathing Cells Remyelinate and Enhance Axonal Conduction in the Demyelinated Dorsal Columns of the Rat Spinal Cord
}

\author{
Toshio Imaizumi, ${ }^{1,2}$ Karen L. Lankford,, ${ }^{1,2}$ Stephen G. Waxman, ${ }^{1,2}$ Charles A. Greer, ${ }^{3}$ and Jeffery D. Kocsis ${ }^{1,2}$ \\ ${ }^{1}$ Department of Neurology, Yale University School of Medicine, New Haven, Connecticut 06510, 2 PVAVEPVA \\ Neuroscience Research Center, Veterans Affairs Medical Center, West Haven, Connecticut 06516, and ${ }^{3}$ Department of \\ Neurosurgery, Yale University School of Medicine, New Haven, Connecticut 06510
}

\begin{abstract}
Olfactory ensheathing cells (OECs), which have properties of both astrocytes and Schwann cells, can remyelinate axons with a Schwann cell-like pattern of myelin. In this study the pattern and extent of remyelination and the electrophysiological properties of dorsal column axons were characterized after transplantation of OECs into a demyelinated rat spinal cord lesion. Dorsal columns of adult rat spinal cords were demyelinated by $x$-ray irradiation and focal injections of ethidium bromide. Cell suspensions of acutely dissociated OECs from neonatal rats were injected into the lesion $6 \mathrm{~d}$ after $\mathrm{x}$-ray irradiation. At 21-25 $d$ after transplantation of OECs, the spinal cords were maintained in an in vitro recording chamber to study the conduction
\end{abstract}

properties of the axons. The remyelinated axons displayed improved conduction velocity and frequency-response properties, and action potentials were conducted a greater distance into the lesion, suggesting that conduction block was overcome. Quantitative histological analysis revealed remyelinated axons near and remote from the cell injection site, indicating extensive migration of OECs within the lesion. These data support the conclusion that transplantation of neonatal OECs results in quantitatively extensive and functional remyelination of demyelinated dorsal column axons.

Key words: olfactory ensheathing cell; transplantation; demyelination; remyelination; dorsal column; spinal cord
Olfactory ensheathing cells (OECs) exhibit a number of unique properties that make them attractive candidates for cell therapies designed to remyelinate demyelinated CNS axons. Although OECs normally do not produce myelin, studies have shown that OECs can myelinate axons both in vitro (Devon and Doucette, 1992) and in vivo (Franklin et al., 1996). OECs are pluripotent, exhibiting properties of both Schwann cells and astrocytes (for review, see Ramon-Cueto and Valverde, 1995). Previous studies have shown that, although transplantation of Schwann cells alone results in myelination of demyelinated or amyelinated spinal cord axons, transplantation of Schwann cells, together with astrocytes, produces much more extensive areas of remyelination (Blakemore and Crang, 1985; Honmou et al., 1996) as well as improvements in the conduction properties of the remyelinated axons (Honmou et al., 1996). Because OECs exhibit both astrocyte-like and Schwann cell-like properties, we reasoned that they might be able to restore axonal conduction properties without cotransplantation of astrocytes. Recent evidence indicating that OECs can promote regeneration of dorsal root ganglion (DRG) axons into the dorsal horn (Ramon-Cueto and Nieto-Sampedro, 1994) and of corticospinal axons across a transection site in the spinal cord (Li et al., 1997) further suggests the possibility that remyelinating cell therapies by using OECs also might facilitate nerve regeneration after injuries involving both axonal demyelination and tran-

Received Feb. 23, 1998; revised May 28, 1998; accepted June 4, 1998.

This work was supported in part by the National Multiple Sclerosis Society, National Institutes of Health (Grant NS10174), and the Medical Research Service, Department of Veterans Affairs.

Correspondence should be addressed to Dr. Jeffery D. Kocsis, Department of Neurology, Yale University School of Medicine Neuroscience Research Center, Veterans Affairs Medical Center, Building 34, West Haven, CT 06516.

Copyright (C) 1998 Society for Neuroscience $\quad 0270-6474 / 98 / 186176-10 \$ 05.00 / 0$ section. This may be significant in light of recent results that indicate some axonal transection in demyelinating lesions of multiple sclerosis (MS) patients (Trapp et al., 1998) (for review, see Waxman, 1998).

It is well established that transplantation of CNS glial cells can result in anatomical (Blakemore and Crang, 1985; Gumpel et al., 1987; Duncan et al., 1988; Rosenbluth et al., 1990; Franklin et al., 1995) as well as electrophysiological (Utzschneider et al., 1994) repair of demyelinated or amyelinated CNS axons. Furthermore, transplantation of purified O2-A progenitor cells alone has been observed to produce significant remyelination of demyelinated spinal cord lesions (Groves et al., 1993), demonstrating that replacement of oligodendrocytes is sufficient to produce some repair of demyelinated CNS lesions. Despite the appeal of oligodendrocyte replacement, immunological considerations in MS patients argue in favor of developing a peripheral source of cells. Peripherally derived Schwann cells or OECs are more accessible than CNS glia and could, at least in principle, be obtained from the host's nerves and thereby avoid the risk of cell rejection or the need for immunosuppression. Furthermore, because MS lesions result from autoimmune attacks on oligodendrocytes, transplanted oligodendrocytes potentially could be at risk for secondary immunological attack, whereas Schwann cells and OECs, expressing different surface antigens, may not.

Although Schwann cells represent a readily accessible source of myelin-forming cells, the apparent requirement for cotransplantation of astrocytes to produce extensive areas of Schwann cell myelination in the CNS indicates a potential limitation to the usefulness of Schwann cells for remyelinating cell therapies (Franklin et al., 1992). Therefore, in this study we have asked whether OECs, which exhibit both astrocyte-like and Schwann cell-like properties, can remyelinate extensive areas of a demy- 

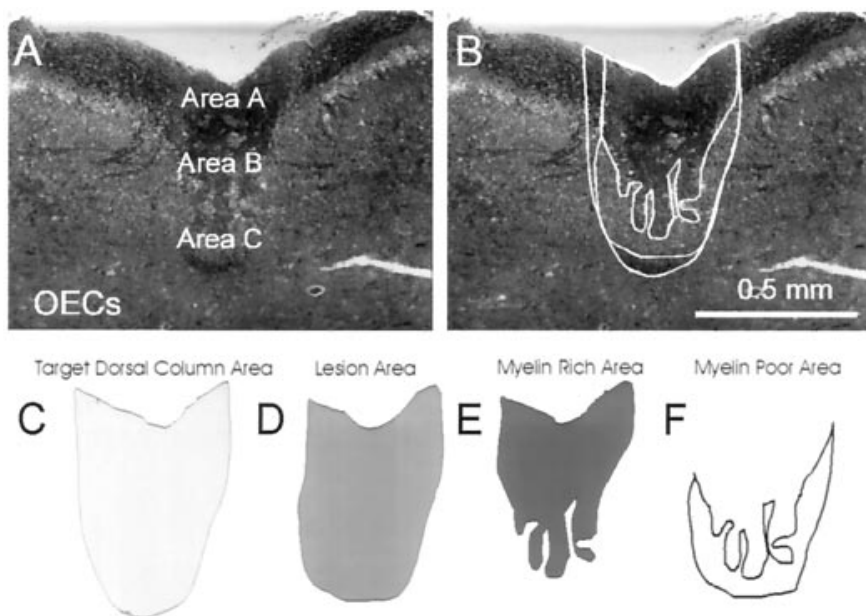

Figure 1. Illustration of regions of the dorsal column identified for subsequent analysis of myelinated axon density and size. $A$, Cross section of an EB-X-lesioned dorsal column with transplanted OECs. Areas $A, B$, and $C$ indicate the dorsomedial, centromedial, and ventromedial regions of the dorsal column, respectively; within these areas the sizes (crosssectional area) of myelinated axons were measured for one representative video field every millimeter along the length of the spinal cord segment that was studied. $B$, Photomicrograph shown in $A$, with the target (central region used for analysis) dorsal column areas, lesioned area, and "myelinrich" and "myelin-poor" areas outlined. Myelinated, demyelinated, and remyelinated areas were identified at low magnification by the staining density. $C-F$, Diagrams showing the cross-sectional areas of the target dorsal column area, demyelinated area, and myelin-rich and myelin-poor areas within the lesion, respectively. These areas were used to calculate the percentage of the target areas lesioned as well as the estimated numbers of remyelinated axons at leach level of the cord. elinated spinal cord lesion and restore electrophysiological properties of the remyelinated axons.

\section{MATERIALS AND METHODS}

Experiments were performed on 20 (6 unoperated controls; 7 EB-Xlesioned, and 7 EB-X-lesioned with transplants) female Wistar rats; one EB-X animal subsequently was eliminated from analysis because of inadequate lesion size.

Induction of demyelination. The induction of the lesion was a modification of the method developed by Blakemore and Patterson (1978) and is described in detail in a previous paper (Honmou et al., 1996). A $40 \mathrm{~Gy}$ surface dose of x-ray irradiation was delivered through a $2 \times 4 \mathrm{~cm}$ opening in a lead shield (4 mm thick) to the spinal cord caudal to T10, using a Siemens Stabilipan radiotherapy machine $(250 \mathrm{kV}, 15 \mathrm{~mA}, 0.5$ $\mathrm{mm} \mathrm{Cu} ; 1 \mathrm{~mm} \mathrm{Al} \mathrm{filters,} \mathrm{SDD} 28 \mathrm{~cm}$, dose rate $220.9 \mathrm{cGy} / \mathrm{min}$; Siemens AG, Erlangen, Germany). At $3 \mathrm{~d}$ after $\mathrm{x}$-ray irradiation, a laminectomy was performed at $\mathrm{T} 11$, and $0.5 \mu \mathrm{l}$ of $0.3 \mathrm{mg} / \mathrm{ml}$ ethidium bromide (EB) in saline was injected unilaterally into the dorsal column at depths of 0.7 and $0.5 \mathrm{~mm}$ at three longitudinal sites $2 \mathrm{~mm}$ apart, for a total of six injections (Felts and Smith, 1992; Honmou et al., 1996).

Cell preparation and transplantation. OECs were separated from neonatal rats ( 2 or $3 \mathrm{~d}$ old), as described previously (Chuah and $\mathrm{Au}, 1993$ ). After the removal of meningeal membranes, olfactory nerve layers were dissected from the olfactory bulb and were cut into pieces and dipped in cold DMEM $\left(4-6^{\circ} \mathrm{C}\right)$ in sterile condition. The tissue blocks of the ONL were triturated through a flame-narrowed glass pipette, filtered through gauze, and then retriturated until single-cell suspensions were obtained. OECs were concentrated at 2.4-3.0/ $\mu$ l in DMEM. The demyelinated rats were anesthetized with ketamine (75 $\mathrm{mg} / \mathrm{kg}$, i.p.) and xylazine $(10$ $\mathrm{mg} / \mathrm{kg}$, i.p.). A dorsal laminectomy was performed at the T11 vertebral level under sterile conditions. A bolus of $0.5 \mu \mathrm{l}$ of the OEC suspension was injected via a glass micropipette into the midpoint of the EB-X lesion 0.7 and $0.5 \mathrm{~mm}$ in depth over a period of $90 \mathrm{sec}$; the micropipette was left in position for $30 \mathrm{sec}$ before slowly being withdrawn. The transplanted site was marked with sterile charcoal.

Electrophysiological recording. Rats were killed under sodium pentobarbital anesthesia ( $75 \mathrm{mg} / \mathrm{kg}$, i.p.) for physiological experiments $21-25 \mathrm{~d}$ after cell injection. The vertebral column between T8 and L2 was removed and placed in cold $\left(4-6^{\circ} \mathrm{C}\right)$ dissecting solution [containing (in mM) 135 choline chloride, 20 choline bicarbonate, $1.0 \mathrm{KCl}, 1.2 \mathrm{KH}_{2} \mathrm{PO}_{4}$, and 90 dextrose]. The spinal cord region between vertebral regions $\mathrm{T} 9$
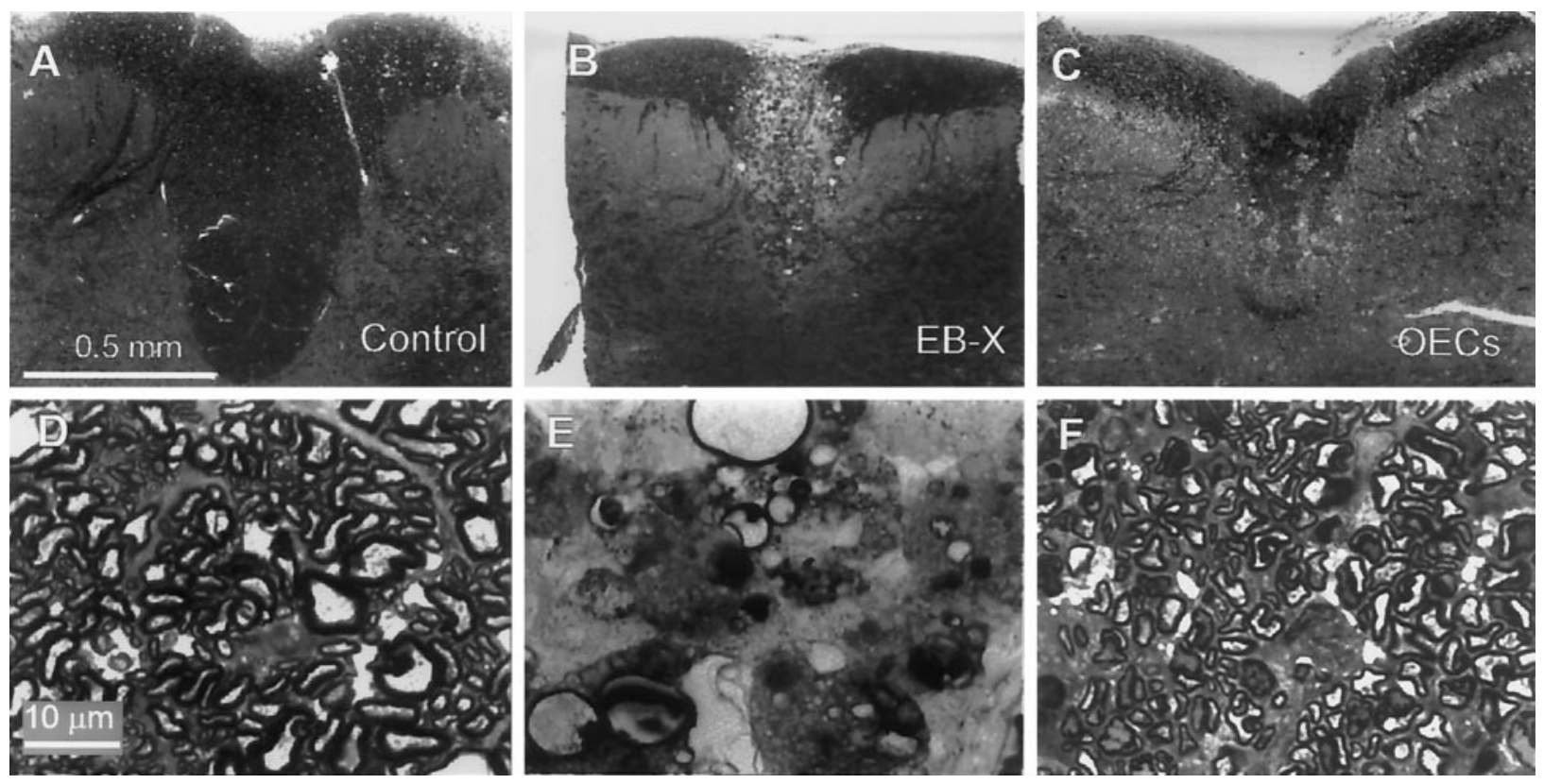

Figure 2. $A-C$, Low-magnification light micrograph of cross sections through dorsal columns from unlesioned control rats $(A)$, EB-X-lesioned rats $(B)$, and animals with EB-X lesions, followed by the transplantation of 30,000 OECs $(C)$. Micrographs $B$ and $C$ show areas near the centers of the lesioned areas. $D-F$, High magnification of images of the centers of the dorsal column shown in the panels directly above these. Few myelinated axons can be detected in the center of the lesioned dorsal column without transplanted OECs, but many can be observed in the lesion with transplanted OECs. Calibration in $A$ refers to $A-C$ and that in $D$ to $D-F$. 

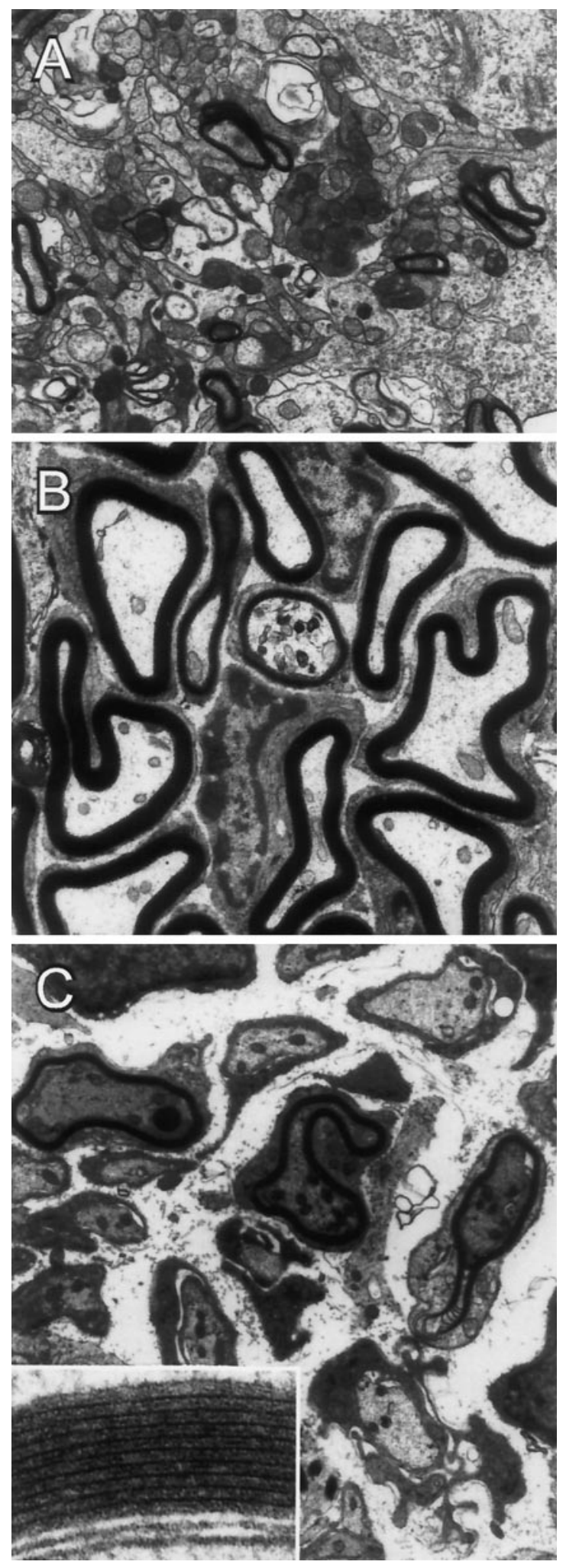

Figure 3. Electron micrographs of representative regions of EB-Xlesioned area of the OEC transplanted dorsal column shown in Figure $2 C$. $A$, Peripheral edge of EB-X lesion. Note primarily nonmyelinated axons. $B, C$, Central areas of the lesion showing examples of remyelinated axons. Intercellular spacing is increased, and most axons appear either to be myelinated $(B)$ or to be in some stage of myelin formation $(C)$. Inset in $C$, High magnification of remyelinated axon showing multilayered membrane structure consistent with that of myelin surrounding the axon. $A-C$, $12,000 \times$ magnification; inset, $170,000 \times$ magnification.
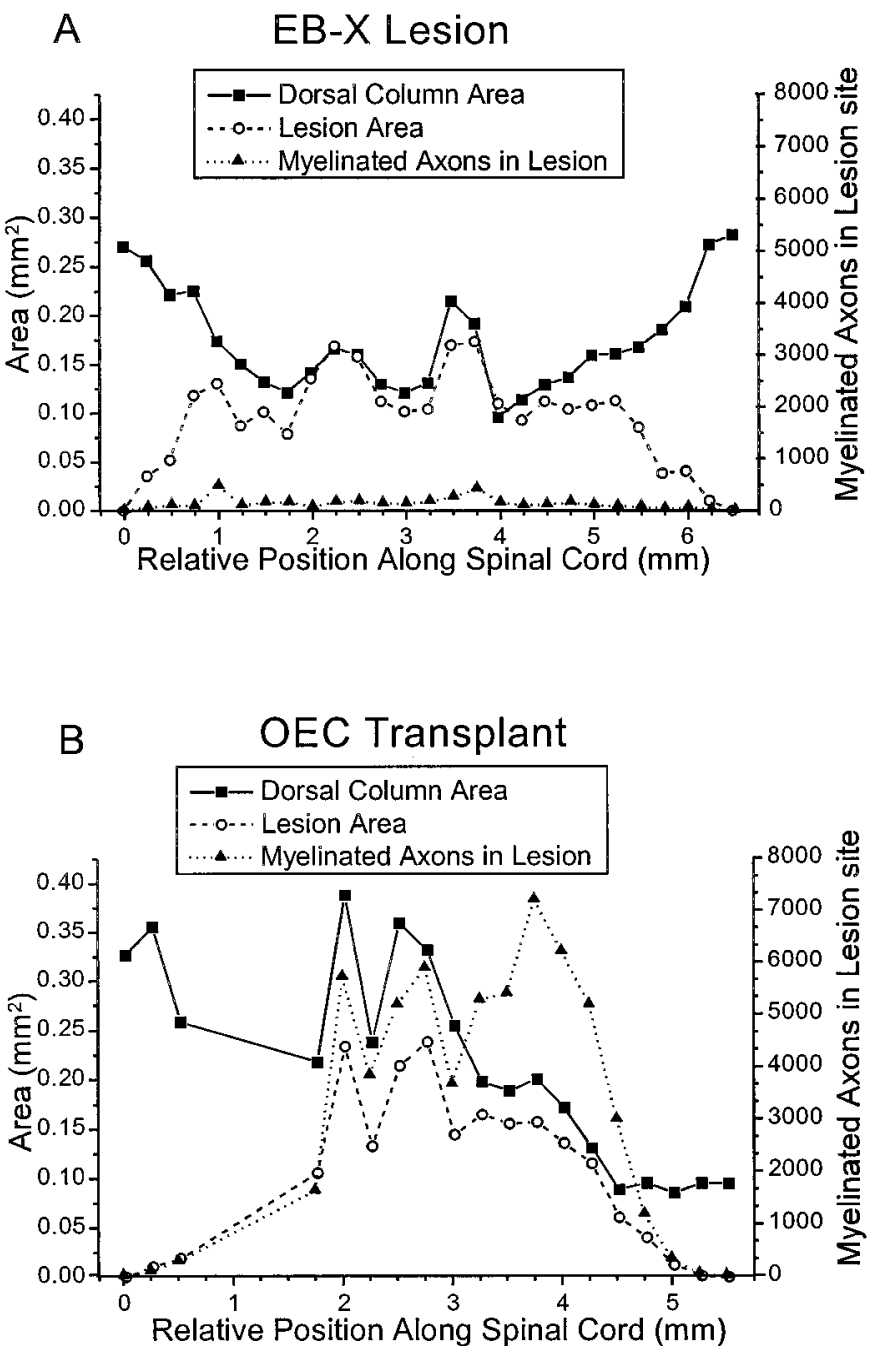

Figure 4. Graphs showing areas of dorsal columns and EB-X-lesioned areas as well as the estimated numbers of remyelinated axons at successive points along the length of the spinal cord for a representative EB-X-lesioned dorsal column $(A)$ and a lesioned dorsal column after 30,000 transplanted OECs $(B)$. The data shown here are for the same lesions shown in Figure $2 B, C$.

and L1 was removed and was pinned in a recording chamber constantly perfused with zero- $\mathrm{Ca}^{2+}$ modified Krebs' solution [containing (in $\mathrm{mM}$ ) $124 \mathrm{NaCl}, 3.0 \mathrm{KCl}, 2.0 \mathrm{MgCl}_{2}, 26 \mathrm{NaH}_{2} \mathrm{CO}_{3}, 1.3 \mathrm{NaHPO}_{4}$, and 10 dextrose] bubbled with $95 \% \mathrm{O}_{2} / 5 \% \mathrm{CO}_{2}$ at a drip rate of $4.0-5.5 \mathrm{ml} / \mathrm{min}$ at room temperature for $30 \mathrm{~min}$. Then $\mathrm{CaCl}_{2}(2.0 \mathrm{mM})$ was added to the solution. At $80 \mathrm{~min}$ after the addition of $\mathrm{CaCl}_{2}$, the electrophysiological recordings were obtained at $26^{\circ} \mathrm{C}$. The Krebs' solution in the chamber gradually was warmed to $36^{\circ} \mathrm{C}$ and maintained at this temperature with a TC-102 temperature controller (Medical Systems, Greenvale, NY ). After a $90 \mathrm{~min}$ incubation at $36^{\circ} \mathrm{C}$, additional recordings were obtained.

The surface of the demyelinated lesion was identified by its translucence and dorsomedial position; the site of OEC injection was identified by the charcoal spot. A bipolar silver wire stimulating electrode was placed on the caudal end of the normal region of the dorsal column. Stimulus amplitude was set $20 \%$ above the strength that elicited a maximal compound action potential (CAP). Stimuli (50 $\mu \mathrm{sec}$ duration) were delivered via a constant current stimulus isolation unit (Master-8, AMPI). The electrical responses were recorded with glass microelectrodes (10 $\mu \mathrm{m}$ tip diameter, 3-10 $\mathrm{M} \Omega$ resistance) filled with $150 \mathrm{~mm}$ $\mathrm{NaCl}$ and positioned within the dorsal columns $2.0-5.0 \mathrm{~mm}$ from the stimulating electrode. CAPs were recorded with a preamplifier (Axoprobe-1, Axon Instruments, Foster City, CA) and displayed on a 


\section{Distribution of Myelinated Axons Sizes in Different Regions of the Dorsal Column}
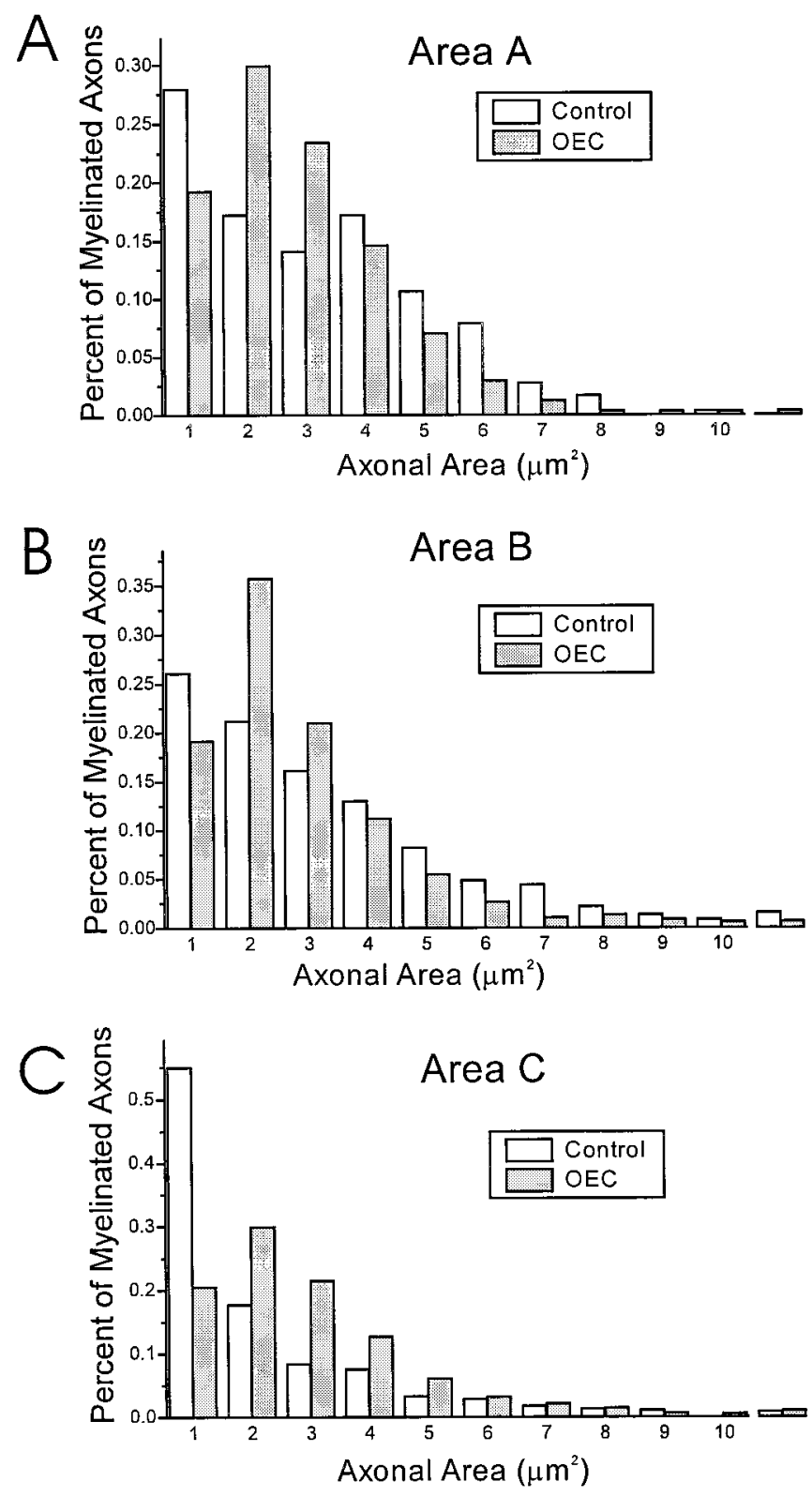

Figure 5. Graphs illustrating the size (area) distribution of myelinated axons in the dorsomedial $(A)$, centromedial $(B)$, and ventromedial $(C)$ of the dorsal columns (designated as areas $A, B$, and $C$ in Fig. $1 B$ ). Each graph shows the percentage of axons in each size category for unoperated controls (white bars) and OEC remyelinated dorsal columns (black bars). Representative fields were analyzed every millimeter along the dorsal column. A minimum of 250 control axons and 1200 OEC remyelinated axons were measured from at least three different experiments to produce each graph. The population distribution for each region is shifted toward larger axon sizes in the OEC remyelinated condition, but the effects were most marked in the ventromedial or corticospinal tract region of the dorsal column.

digital oscilloscope (4094C, Nicolet Instruments, Madison, WI). CAP amplitudes were measured from peak to peak and were compared with the control CAP amplitude. All data were expressed as mean \pm SD.

Histological examination. After electrophysiological recordings were made, spinal cord tissue was fixed for $24 \mathrm{hr}$ in $2 \%$ paraformaldehyde plus
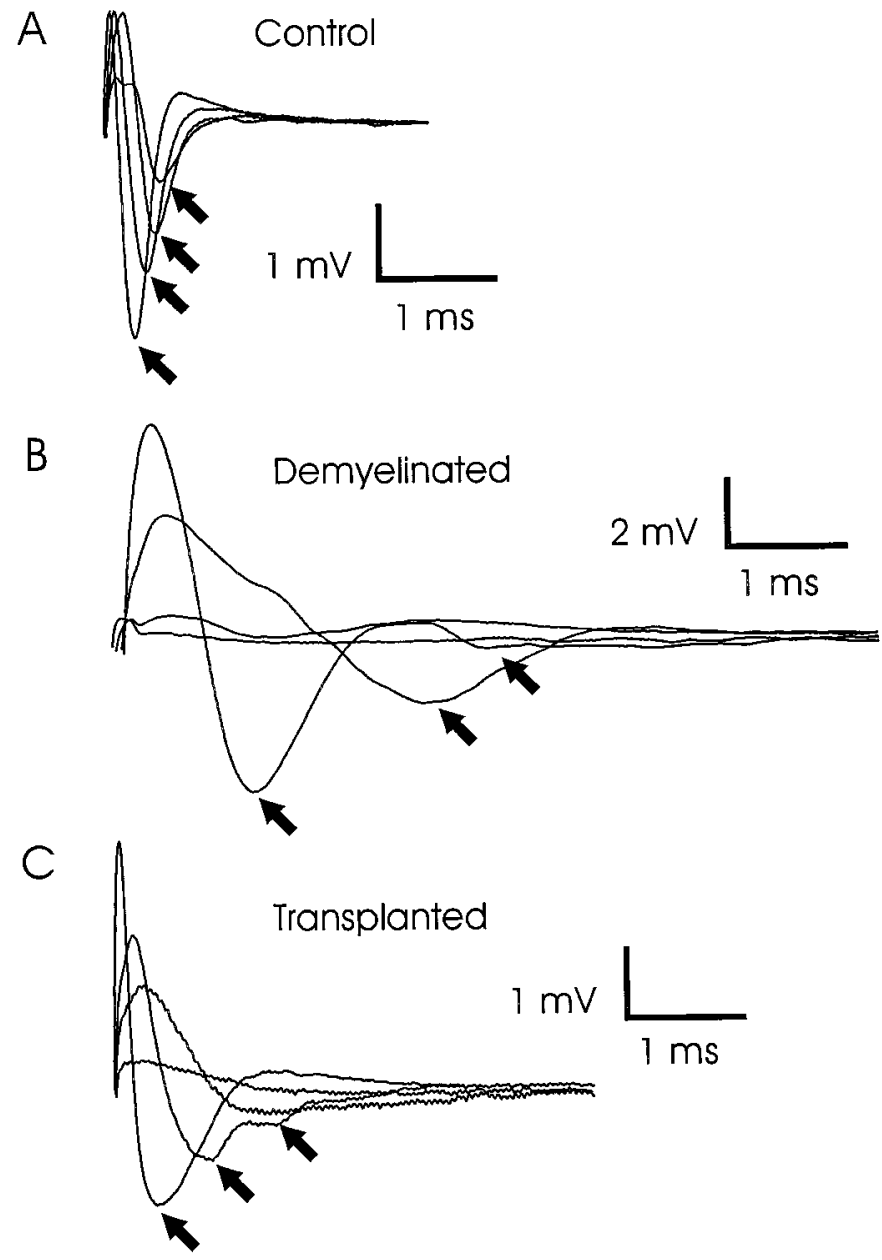

Figure 6. Field potential recordings from control $(A)$, demyelinated $(B)$, and transplanted $(C)$ dorsal columns. Compound action potentials were recorded at four points at $1 \mathrm{~mm}$ increments (arrows) along the dorsal columns. Note the increase in latency for recordings obtained from the demyelinated axons and the decrease in latency for those obtained from the dorsal columns with OEC transplantation.

$2 \%$ glutaraldehyde (w/v in 0.14 m Sorensen's buffer). Tissue was washed three times, stored overnight in buffer, and cut into $2 \mathrm{~mm}$ segments. Then spinal cord segments were post-fixed with $1 \%$ osmium tetroxide (Polysciences, Warrington, PA) for $4 \mathrm{hr}$ and embedded in Epox-812 (Ernest Fullam, Latham, NY). Tissue was serially sectioned on an ultramicrotome, and $1 \mu \mathrm{m}$ sections were collected every $0.25 \mathrm{~mm}$. Finally, the sections were counterstained with $0.5 \%$ methylene blue $/ 0.5 \%$ azure II in $0.5 \%$ borax.

To assess the degree of remyelination produced by transplanted OEC cells, we first measured cross-sectional areas of the medial aspect of the dorsal column within each section, the demyelinated region of the dorsal column, and any "myelin-rich" areas with a Nikon Microphot microscope with a $4 \times$ lens coupled to a MCI CCD2 video camera and interfaced to an Image 1 processing system. Regions showing different degrees of myelination are readily apparent at this magnification by their staining density (see Fig. 1). Then the percentage of the target region that was demyelinated and the sizes of the regions within the lesion showing relatively high and low percentages of myelination were calculated. The numbers of myelinated axons in 3-10 representative fields in the "myelinrich" (i.e., remyelinated) and "myelin-poor" (i.e., demyelinated) regions of the lesion were counted at $100 \times$ magnification. Small nonmyelinated axons were more difficult to identify with this type of preparation, and their numbers were not included in this analysis. The degree of remyelination at each level of the spinal cord was calculated by using the formula: 


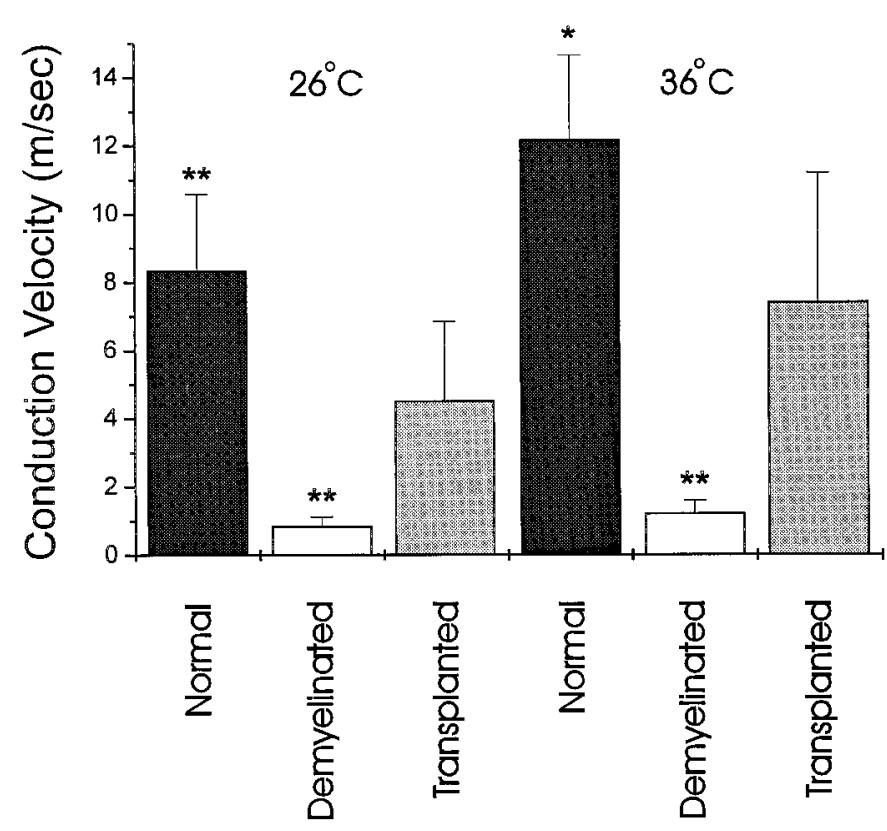

Figure 7. Summary data of conduction velocity for normal $(n=6)$, demyelinated $(n=7)$, and remyelinated $(n=6)$ dorsal column axons at $26^{\circ} \mathrm{C}$ (left) and $36^{\circ} \mathrm{C}$ (right). Conduction velocity of the demyelinated axons was reduced as compared with control at both temperatures $(p<$ 0.00001 , Student's $t$ test). Conduction velocity of the transplant group was increased as compared with the demyelinated axons but decreased as compared with the normal axons. Significance levels are indicated by asterisks $\left({ }^{*} p<0.05 ;{ }^{*} p<0.01\right.$; shown as comparisons of normal or demyelinated groups to the transplant group).

$$
N_{\mathrm{X}}=\left(M_{\mathrm{R}} \cdot A_{\mathrm{R}}\right)+\left(M_{\mathrm{P}} \cdot A_{\mathrm{P}}\right),
$$

where $N_{\mathrm{x}}=$ estimated number of myelinated axons at position $X, M_{\mathrm{R}}=$ average density of myelinated axons in the myelin-rich region of the lesion, $M_{\mathrm{P}}=$ average density of myelinated axons in the myelin-poor region of the lesion, $A_{\mathrm{R}}=$ area of the myelin-rich region of the lesion, and $A_{\mathrm{P}}=$ area of myelin-poor region of the lesion.

Calculated numbers of myelinated axons at each level then were integrated across distance to establish the total amount of axon length that was remyelinated and expressed in axon millimeters. Estimates of the percentage of axons remyelinated were obtained by dividing the numbers of axon millimeters remyelinated by the product of the area of the lesion and the average myelinated axon density in unlesioned dorsal columns. The total sizes of the lesioned areas also were calculated by integrating the size of the lesion across distance. Lengths of the lesioned and remyelinated areas were determined from the distance between the first and last sections that showed detectable areas of complete demyelination and the distance between sections with at least 200 remyelinated axons.

To assess the types of fibers remyelinated by OECs, we measured the cross-sectional areas of myelinated and unmyelinated axons in one video field each for three different anatomical regions of the dorsal column. Cross-sectional areas of all myelinated axons were measured in one video field each in the dorsomedial, centromedial, and ventromedial or corticospinal tract region of the dorsal column at $1 \mathrm{~mm}$ intervals along the dorsal column (see Fig. 1A). Myelinated axon size data for each region were pooled for all animals within each treatment condition. The numbers of axons in each $1 \mu \mathrm{m}^{2}$ size (area) category were determined for control and remyelinated axons, and the size distribution of myelinated axons was compared by using a $\chi^{2}$ test.

For some experiments, ultrathin sections also were collected from regions of the spinal cord showing extensive remyelination. Sections were stained with uranyl acetate and lead citrate and examined with a Zeiss EM902A electron microscope (Oberkochen, Germany) operating at 80 $\mathrm{kV}$. Electron micrographs of remyelinated areas were taken at 7000 and $50,000 \times$ magnification.

\section{RESULTS}

\section{Remyelination of the EB-X lesion after OEC transplantation}

Histological examination of spinal cords confirmed that the EB injection and x-ray irradiation of the spinal column resulted in extensive demyelination of the central region of the dorsal columns, with an average of $90 \pm 3 \%$ ( $n=4$ animals) of the target area demyelinated at the widest part of the lesion. To avoid the possibility that large numbers of spared axons could confound interpretation of the conduction properties of demyelinated axons, we did not include animals in any subsequent analysis if $\leq 75 \%$ of the target dorsal column area was lesioned at the widest point in the lesion. EB-X lesions resulted in demyelinating lesions typically $5-7 \mathrm{~mm}$ in total length, with an average distance of $6 \pm$ $0.25 \mathrm{~mm}(n=8$ animals $)$. Total volumes of the lesioned areas averaged $0.9 \pm 0.3 \mathrm{~mm}^{3}(n=4$ animals $)$ for lesioned areas without transplanted cells and $1.0 \pm 0.2 \mathrm{~mm}^{3}$ ( $n=4$ animals) for lesioned areas with transplanted OECs, or an estimated average loss of 120,000 $\pm 20,000 \mathrm{~mm}$ ( $n=4$ animals) of myelinated axon length for each lesion. In control lesions without cell transplants, demyelination of axons within the lesioned area was essentially complete, with $<2 \%$ of axons within the lesioned area either being spared demyelination or spontaneously remyelinated.

Transplantation of OECs into previously demyelinated regions of the dorsal column resulted in remyelination of large areas of the EB-X lesion, near the site of cell transplantation at the approximate center of the lesion, and for $2-3 \mathrm{~mm}$ rostral and caudal to the midpoint. Large myelin-rich areas were detected readily in lesions containing transplanted OECs (Fig. 2C), but not in lesioned dorsal columns into which only culture medium was injected (Fig. 2B). Migration of myelin-forming cells was extensive, with at least 200 remyelinated axons being detected across distances of 4-6 $\mathrm{mm}$ along the spinal cord (average $5.0 \pm 0.5 \mathrm{~mm}$; $n=4$ animals).

Examination of dorsal column axons at the electron microscopic level revealed myelination patterns consistent with interpretations at the light microscopic level of myelin-rich and myelinpoor areas within the transplanted spinal cords. At the ultrastructural level, peripheral areas of OEC transplanted lesions that were categorized as myelin-poor contained primarily densely packed nonmyelinated axons, with small numbers of scattered myelinated axons and cell bodies of undetermined type (Fig. $3 A$ ). Little extracellular space was observed between axons in these areas. In the more central portions of the lesions, however, the majority of axons either exhibited the characteristic features of myelination, with multiple layers of closely apposed membrane surrounding the axons (Fig. $3 B, C$ ), or appeared to be in earlier stages of myelin formation (Fig. 3C). The relative amounts of extracellular space also tended to be larger in regions of apparent remyelination. Although we occasionally observed structures consistent with astrocytic processes in transplanted regions, astrocyte-like cells were not prominent features of lesioned dorsal columns.

The total amount of remyelination observed in lesions with transplanted OECs was significantly greater than that observed in control lesions without transplanted cells (Fig. 4). Transplantation of 24,000-30,000 OECs resulted in the remyelination of $\sim 22,000 \pm 3000 \mathrm{~mm}(n=4$ animals $)$ of total axon length as compared with $2000 \pm 1000$ axon $\mathrm{mm}(n=4$ animals $)$ either spared or spontaneously remyelinated in control lesions $(p<$ 0.001 , unpaired Student's $t$ test). Using the density of myelinated 


\section{A Conduction Distance $\left(26^{\circ} \mathrm{C}\right)$}

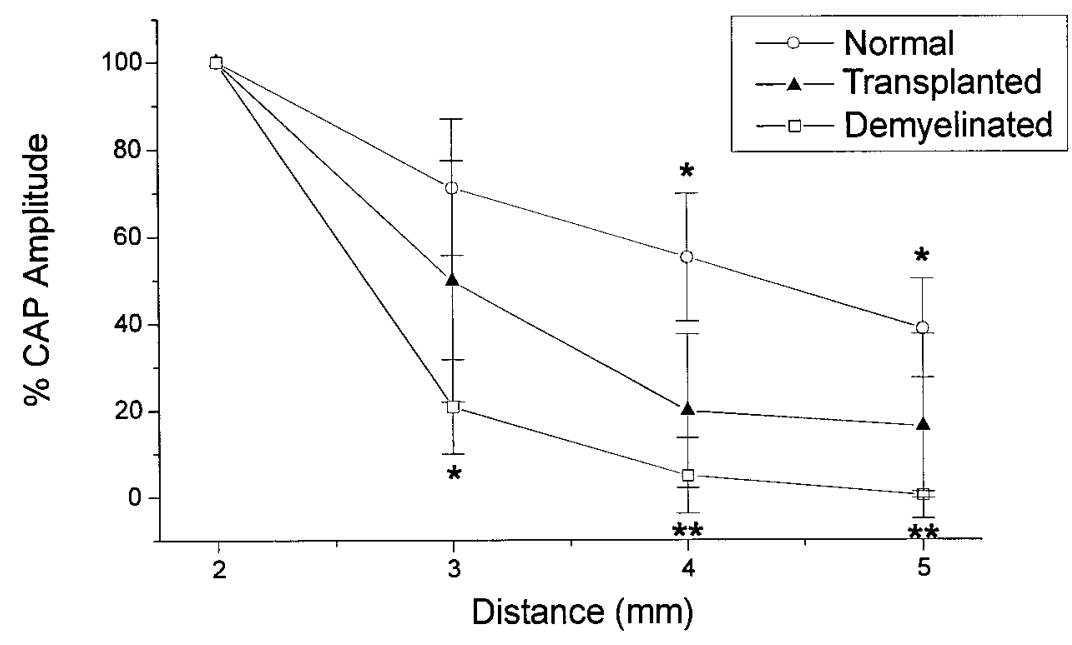

B Conduction Distance $\left(36^{\circ} \mathrm{C}\right)$

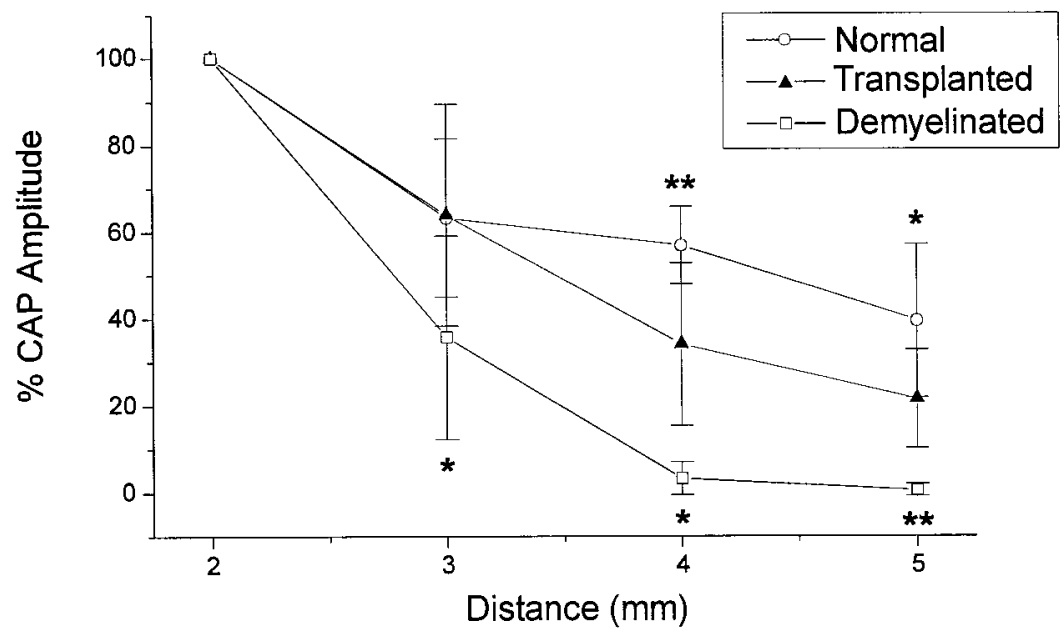

Figure 8. Amplitude decrement with conduction distance for normal $(n=6)$, demyelinated $(n=7)$, and transplanted $(n=6)$ dorsal columns at $26^{\circ} \mathrm{C}(A)$ and $36^{\circ} \mathrm{C}(B)$. Note that axons in the transplant group show less amplitude decrement with increasing conduction distance than axons in the EB-X lesion condition; $p$ value notation and $n$ values are the same as described in Figure 7. axons in unlesioned spinal cords as a reference, these results indicate that transplantation of OECs results in remyelination of $\sim 17 \pm 2 \%$ of demyelinated axons, compared with $1.8 \pm 0.3 \%$ of axons spared or remyelinated in control lesions without transplanted cells. Because dorsal column areas tend to decrease in EB-X-lesioned dorsal columns and increase in lesions with transplanted OECs, these figures probably represent an underestimate of the difference between control lesions and lesions with transplanted OECs.

OECs appeared to be capable of remyelinating axons in all areas of the medial aspect of the dorsal column, although individual experiments often showed that remyelination was localized to a given portion of the dorsal columns. The overall shapes of the myelin-rich areas within a given lesion also tended to be similar in sections separated by a $1 \mathrm{~mm}$ or more, implying that OECs can migrate more readily along the parallel rather than the perpendicular axonal axis and that small differences in positioning of the injection site could be responsible for differences in the subsequent patterns of remyelination. Although some axons in all size categories were remyelinated in lesions with OEC transplants, size distributions of remyelinated axons in the OEC transplant condition appeared to be skewed toward larger diameter axons when compared with myelinated axon sizes for similar regions of control dorsal columns ( $p<0.005, \chi^{2}$ test) (Fig. 5). Differences in the diameter of myelinated axons were particularly apparent in the corticospinal tract region of the dorsal column, where the average size (area) of myelinated axons was increased from $1.72 \pm 0.09 \mu \mathrm{m}^{2}$ in control spinal cords to $2.55 \pm 0.06 \mu \mathrm{m}^{2}$ in the OEC remyelinated condition.

\section{Enhanced axonal conduction after remyelination by OECs}

Consistent with histological evidence for extensive demyelination of EB-X-lesioned dorsal column and substantial remyelination by transplanted OECs, field potential recordings revealed significant reductions in conduction velocity and frequency-response properties in EB-X-lesioned dorsal columns, whereas conduction velocities and frequency-response properties in lesioned dorsal columns with transplanted OECs were more similar to control unlesioned dorsal columns. Figure $6 A$ shows four superimposed 


\section{A Paired-Pulse Stimuli $\left(26^{\circ} \mathrm{C}\right)$}

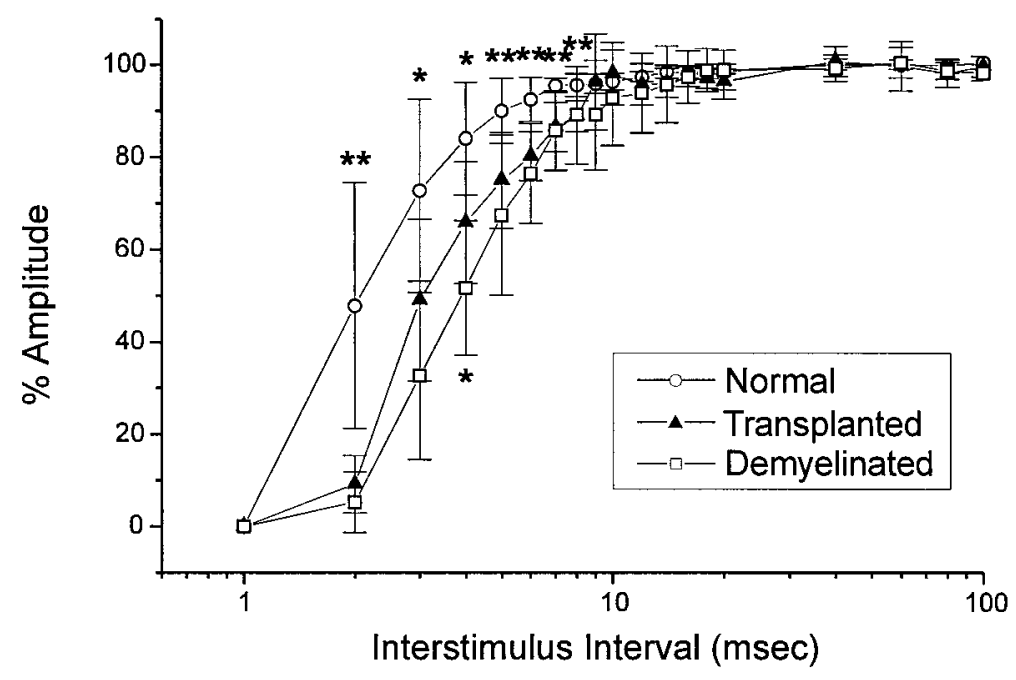

B Paired-Pulse Stimuli $\left(36^{\circ} \mathrm{C}\right)$

Figure 9. Amplitude recovery for the second of two stimuli at varying interstimulus intervals for normal, demyelinated, and transplanted dorsal columns at $26^{\circ} \mathrm{C}(A)$ and $36^{\circ} \mathrm{C}(B)$. There is less amplitude decrement for the remyelinated axons than for the demyelinated axons. $p$ value notation and $n$ values are the same as described in Figure 7 and indicate the comparison between the normal or demyelinated condition to the transplant group.

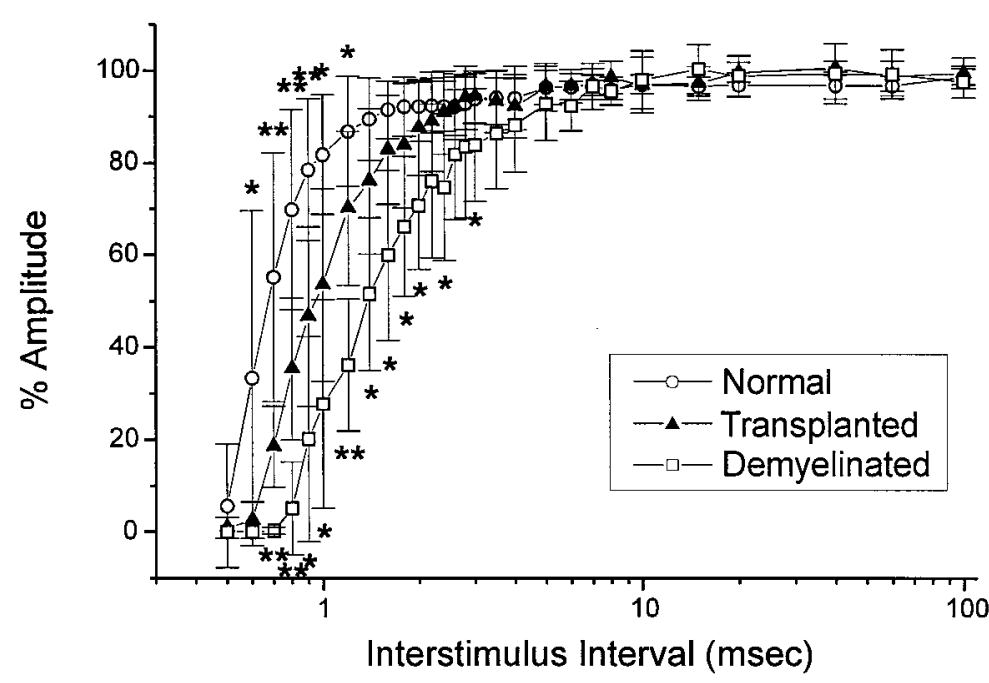

larger amplitude decrements with increased conduction distance as compared with control dorsal column recordings, indicating extensive conduction block (Fig. 8). The responses from the region remyelinated by OECs displayed less amplitude decrement with increasing conduction distance (Fig. 8, triangles); unlike the recordings from the demyelinated areas in which virtually no response was present at $4 \mathrm{~mm}$ from the recording site, the remyelinated axons were able to conduct at least $5 \mathrm{~mm}$ into the lesion, suggesting a reduction of conduction block.

Paired stimulus pulses applied at various intervals were used to study the recovery properties of the axons; interstimulus intervals ranged from 1 to $100 \mathrm{msec}$ at $26^{\circ} \mathrm{C}$ and from 0.5 to $100 \mathrm{msec}$ at $36^{\circ} \mathrm{C}$. CAP amplitude recovery was reduced and delayed in demyelinated regions but improved after the OEC transplantation at both temperatures (Fig. 9). The ability of dorsal column axons to transmit trains of action potentials was examined via frequen- with increasing conduction distance. The CAPs from the demyelinated dorsal columns showed larger increases in latency and
$(4.52 \pm 2.32 \mathrm{~m} / \mathrm{sec}, n=6 ; p<0.006, t$ test $)$ and $36^{\circ} \mathrm{C}(7.40 \pm 3.78$ $\mathrm{m} / \mathrm{sec}, n=6 ; p<0.006)$ as compared with demyelinated axons. A summary of these results is shown graphically in Figure 7.

In normal dorsal columns the amplitude of the CAP decreased 
A $\quad \operatorname{Train}\left(26^{\circ} \mathrm{C}\right)$
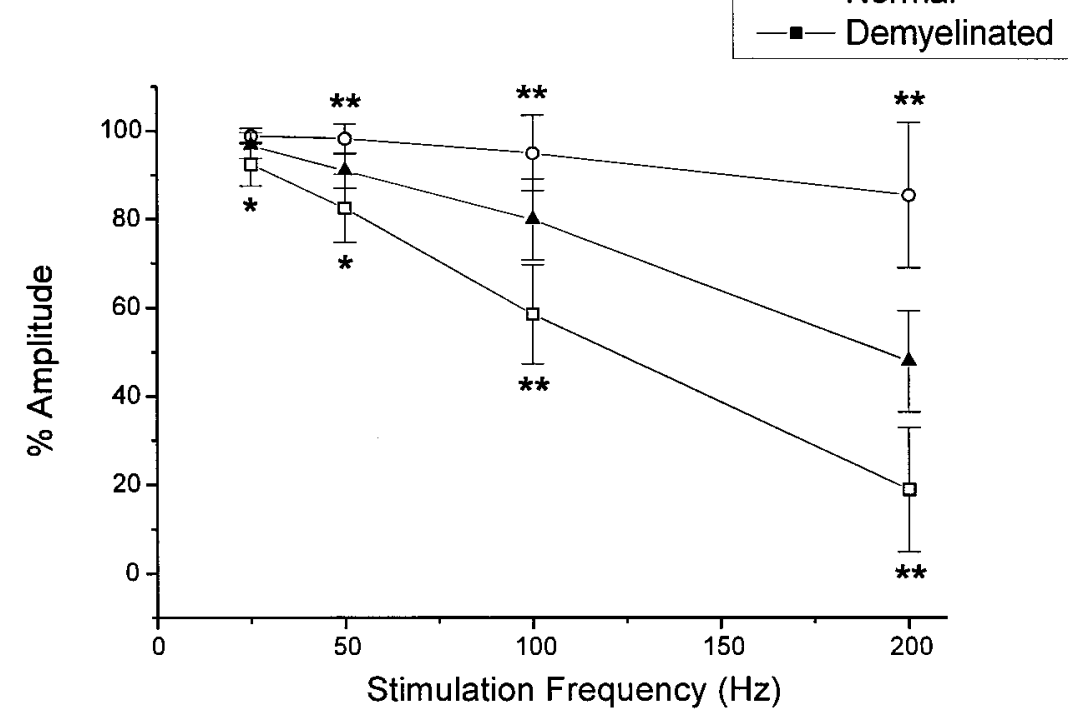

B $\quad \operatorname{Train}\left(36^{\circ} \mathrm{C}\right)$
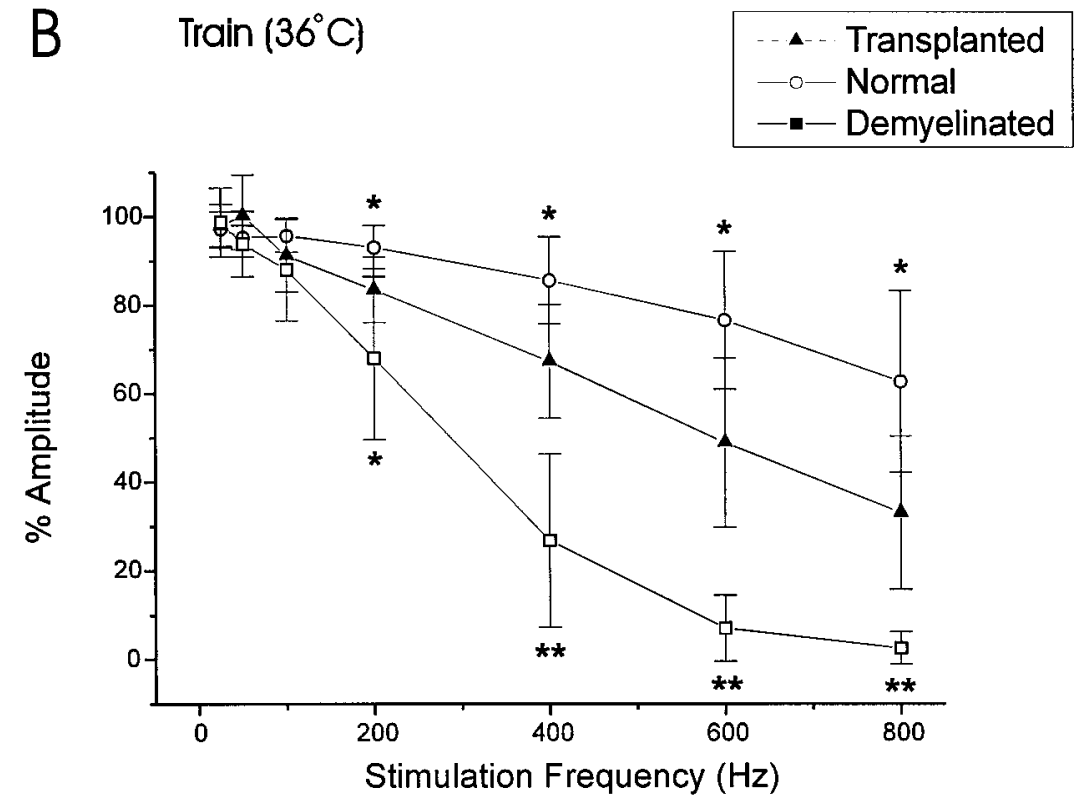

Figure 10. Frequency-response properties of normal, demyelinated, and transplanted dorsal columns at $26^{\circ} \mathrm{C}(A)$ and $36^{\circ} \mathrm{C}(B)$. The demyelinated axons display considerable reduction in their ability to follow high-frequency stimulation, but the remyelinated axons are able to follow higher frequencies of stimulation. Comparisons of responses between normal or demyelinated groups are shown related to the transplant group. $p$ value notation and $n$ values are the same as described in Figure 7. cy-response analysis, using $25-200 \mathrm{~Hz}$ stimulus trains $(0.5 \mathrm{sec}$ duration) at $26^{\circ} \mathrm{C}$ and $25-800 \mathrm{~Hz}$ at $36^{\circ} \mathrm{C}$. Control dorsal column axons displayed a progressive amplitude decrement to $10 \%$ at $26^{\circ} \mathrm{C}(200 \mathrm{~Hz})$ and $30 \%$ of initial amplitude at $36^{\circ} \mathrm{C}(800 \mathrm{~Hz})$ (Fig. 10). At both temperatures the demyelinated axons showed nearly complete conduction block at high frequencies. This defect in conduction was reversed partially, however, by OEC transplantation, and the OEC-remyelinated axons showed considerable restoration in their ability to follow high-frequency stimulation (Fig. 10).

\section{DISCUSSION}

In the present study we demonstrate that transplantation of OECs into EB-X demyelinated adult rat spinal cords results in relatively extensive remyelination of demyelinated axons and recovery of electrophysiological function. Histological, ultrastructural, and electrophysiological data all support the idea that transplanted
OECs can produce substantial repair of demyelinated spinal cord axons. The pattern of remyelination produced by transplanted OECs was very similar to that produced by cotransplantation of neonatal Schwann cells and astrocytes (Honmou et al., 1996), i.e., relatively thick myelin with large cytoplasmic and nuclear regions surrounding remyelinated axons, suggesting that a single OEC remyelinates a single axonal segment. A notable difference between remyelination by Schwann cells and OECs was that transplantation of neonatal Schwann cells without astrocyte cotransplantation resulted in only small restricted areas of remyelination, whereas OECs were capable of migrating and remyelinating axons across distances of several millimeters without the need for transplantation of additional cell types. This observation suggests that astrocytes may produce a signal that induces the migration of Schwann cells, as suggested by Honmou et al. (1996). Indeed, preliminary studies in our laboratory suggest that injections of 
Schwann cells cultured in conditioned astrocyte medium display more extensive myelination than Schwann cells alone.

OECs have a number of unique properties that could account for their ability to remyelinate axons in the CNS. As summarized by Doucette and colleagues (Doucette, 1991, 1993; Doucette and Devon, 1993, 1994) and recently reviewed by Ramon-Cueto and Valverde (1995), these cells share properties of both Schwann cells and astrocytes. They express p7S ${ }^{\text {NGFR }}$, L1, PSA-NCAM, laminin, central-GFAP, myelin basic protein, neuropeptide $\mathrm{Y}$, and glial nexin and may contribute to the secretion of NGF. In olfactory nerve they typically do not express a myelinating phenotype and appear morphologically similar to astrocytes (Doucette and Devon, 1993). However, the addition of OECs to cultures of DRG neurons results in their development of a well defined myelinating phenotype similar to that observed by Schwann cells (Devon and Doucette, 1992; Ramon-Cueto and Nieto-Sampedro, 1993). Moreover, Franklin et al. (1996) demonstrated that the injection of OECs into the demyelinated rat spinal cord results in remyelination. Although one possibility is that transplantation of OECs may result in in vivo differentiation of both astrocyte-like and Schwann cell-like cells that interact to provide conditions appropriate for relatively extensive migration and myelination, we did not see extensive evidence for the presence of astrocytes in the lesioned area. Consequently, we tend to favor the possibility that the OECs intrinsically possess greater myelinating and migratory potential than Schwann cells.

In the present study we have examined the electrophysiological properties of the OEC-induced remyelinated axons. The results indicate that the conduction velocity of the remyelinated axons is restored toward normal values and that the axons are capable of following relatively high-frequency stimulation trains. Moreover, impulses conduct a greater distance along their longitudinal axis in the dorsal columns of the remyelinated axons than in the demyelinated axons, suggesting that conduction block is overcome in the axons remyelinated by OEC transplantation. These data suggest that a sufficient nodal $\mathrm{Na}^{+}$channel density is present in the OEC remyelinated axons to establish relatively normal conduction.

Examination of the morphology of the remyelinated spinal cord indicates substantial structural differences as compared with normal spinal cord. The density of axons is lower in the remyelinated dorsal columns; this results from the increased space occupied by the cell bodies and nuclei of the OECs as compared with oligodendrocytes. In this regard, the general morphological appearance of the remyelinated axon regions in the dorsal columns is reminiscent of peripheral nerve. Extracellular microelectrode recordings from sciatic nerve are of relatively low amplitude as compared with recordings in CNS white matter. The larger extraaxonal space of both peripheral nerve and the reconstructed spinal cord white matter could provide a greater extra-axonal current pathway that would lower the extracellular current density recorded by the extracellular microelectrode tip, resulting in a reduced amplitude of the response. The reduced amplitude of the CAP of the remyelinated axons also likely reflects a reduction in the number of axons conducting action potentials.

Recently, Li et al. (1997) reported that injecting a suspension of OECs at the site of a lesion in the corticospinal tract resulted in the extension of transected corticospinal axons through the lesion. The OECs cells adopted a myelinating phenotype with individual axons that persisted through the lesion site. Those animals with successful axon extension through the lesion also exhibited a restoration of directed forepaw reaching. Moreover, recent stud- ies indicate that some axonal transection can occur in MS patients (Trapp et al., 1998) (for review, see Waxman, 1998). Given that in contusive spinal cord injury and perhaps in some MS patients both axonal transection and demyelination can occur, the OEC may offer a unique opportunity to address both types of pathologies by a monocellular approach.

\section{Concluding remarks}

Our current data indicate that OECs may be good candidates for remyelinating cell therapies. OECs migrate extensively and remyelinate a significant fraction of demyelinated axons without the need for cotransplanting other cell types. Although we cannot rule out the possibility of unusually long internodal distances for OEC remyelinated axons, the amount of remyelinated axon length relative to the numbers of transplanted OECs also suggests that at least some OECs divide after transplantation. Ultrastructural evidence of ongoing myelination at 4 weeks after transplantation implies that even greater repair may be possible with longer recovery times, although in this model it was necessary to terminate the experiment after a relatively short time period to minimize any confounding effects of spontaneous remyelination. Notable, in addition to histological evidence for remyelination by OECs, the transplantation of OECs into demyelinated lesions also results in improved conduction properties.

\section{REFERENCES}

Blakemore WF, Crang AJ (1985) The use of cultured autologous Schwann cells to remyelinated areas of persistent demyelination in the central nervous system. J Neurol Sci 70:207-223.

Blakemore WF, Patterson RC (1978) Suppression of remyelination in the CNS by X-irradiation. Acta Neuropathol (Berl) 42:105-113.

Chuah MI, Au C (1993) Cultures of ensheathing cells from neonatal rat olfactory bulbs. Brain Res 601:213-220.

Devon R, Doucette R (1992) Olfactory ensheathing cells myelinate dorsal root ganglion neurites. Brain Res 589:175-179.

Doucette R (1991) PNS-CNS transitional zone of the first cranial nerve. J Comp Neurol 312:451-466.

Doucette R (1993) Glial cells in the nerve fiber layer of the main olfactory bulb of embryonic and adult mammals. Microsc Res Tech 24:113-130.

Doucette R, Devon R (1993) Olfactory ensheathing cells: factors influencing the phenotype of these glial cells. In: Biology and pathology of astrocyte-neuron interactions (Federoff F, Juurlink B, Doucette R, eds), pp 117-124. New York: Plenum.

Doucette R, Devon R (1994) Media that support the growth and differentiation of oligodendrocytes do not induce olfactory ensheathing cells to express a myelinating phenotype. Glia 10:296-310.

Duncan ID, Hammang JP, Jackson KF, Wood PM, Bunge RP, Langford L (1988) Transplantation of oligodendrocytes and Schwann cells into the spinal cord of the myelin-deficient rat. J Neurocytol 17:351-360.

Felts PA, Smith KJ (1992) Conduction properties of central nerve fibers remyelinated by Schwann cells. Brain Res 574:178-192.

Franklin RJM, Crang AJ, Blakemore WF (1992) Type I astrocytes fail to inhibit Schwann cell remyelination of CNS axons in the absence of cells of the 0-2A lineage. Dev Neurosci 14:85-92.

Franklin RJM, Bayley SA, Blakemore WF (1995) Differentiation of the $\mathrm{O}-2 \mathrm{~A}$ progenitor cell line CG-4 into oligodendrocytes and astrocytes following transplantation into glia-deficient areas of CNS white matter. Glia 13:39-44.

Franklin RJ, Gilsson JM, Franceschini IA, Barnett SC (1996) Schwann cell-like myelination following transplantation of an olfactory bulbensheathing cell line into areas of demyelination in the adult CNS. Glia 17:217-224.

Groves AK, Barnett SC, Franklin RJ, Crang AJ, Mayer M, Blakemore WE, Noble M (1993) Repair of demyelinated lesions by transplantation of purified O-2A progenitor cells. Nature 362:453-455.

Gumpel M, Lachapelle F, Gansmuller A, Baulac M, Baron-Van Evercooren A, Baumann N (1987) Transplantation of human embryonic oligodendrocytes into shiverer brain. Ann NY Acad Sci 495:71-85. 
Honmou O, Felts PA, Waxman SG, Kocsis JD (1996) Restoration of normal conduction properties in demyelinated spinal cord axons in the adult rat by transplantation of exogenous Schwann cells. J Neurosci 16:3199-3208.

Li Y, Field PM, Raisman G (1997) Repair of adult rat corticospinal tract by transplants of olfactory ensheathing cells. Science 277:2000-2002.

Ramon-Cueto A, Nieto-Sampedro M (1993) In vitro enfolding of olfactory neurites by $\mathrm{p} 75 \mathrm{NGF}$ receptor positive ensheathing cells from adult rat olfactory bulb. Eur J Neurosci 5:1172-1180.

Ramon-Cueto A, Nieto-Sampedro M (1994) Regeneration into the spinal cord by transected dorsal root axons is promoted by ensheathing glia cells. Exp Neurol 127:232-244.

Ramon-Cueto A, Valverde F (1995) Olfactory bulb ensheathing glia: a unique cell type with axonal growth-promoting properties. Glia 14:163-173.

Rosenbluth J, Hasegawa M, Shirasaki N, Rosen CL, Liu Z (1990) Myelin formation following transplantation of normal fetal glia into myelindeficient rat spinal cord. J Neurocytol 19:718-730.

Trapp BD, Peterson J, Ransohoff RM, Rudick R, Mork S, Bo L (1998) Axonal transection in the lesions of multiple sclerosis. N Engl J Med 338:278-285.

Utzschneider DA, Archer DR, Kocsis JD, Waxman SG, Duncan ID (1994) Transplantation of glia cells enhances action potential conduction of amyelinated spinal cord axons in the myelin-deficient rat. Proc Natl Acad Sci USA 91:53-57.

Waxman SG (1998) Demyelinating diseases: new pathological insights, new therapeutic targets. N Engl J Med 338:323-325. 